\title{
STUDIES ON MYOCARDIAL METABOLISM. V. THE EFFECTS OF LANATOSIDE-C ON THE METABOLISM OF THE HUMAN HEART ${ }^{1}$
}

\author{
By J. M. BLAIN, E. E. EDDlEMAN, A. SIEGEL, AND R. J. BING \\ (From the Departments of Experimental Medicine and Clinical Physiology, The Medical College \\ of Alabama, Birmingham, Alabama, and the Veterans' Administration Hospital, \\ Birmingham, Ala.)
}

(Submitted for publication September 6, 1955; accepted November 16, 1955)

Since 1785 when Withering undertook the first systemic study of the effects of digitalis, a large amount of data has been accumulated describing its pharmacological effects. Despite intensive study, the underlying mechanism responsible for the beneficial results in patients with heart failure remains obscure. Although digitalis in small doses may affect the heart rate through its vagal effect, it is agreed that the predominant action of this drug is on the heart muscle directly (1). In addition, the cardiac glycosides also influence the ionic gradient between the muscle cell and the surrounding medium (2-8). Its effect on oxygen consumption and foodstuff utilization of the heart has been repeatedly investigated in the heart-lung preparation, in tissue slices and in tissue homogenates (9). The reader is here referred to the review article of Wollenberger (10). However, the abundance of conflicting results suggests that cardiac glycosides may exhibit different metabolic defects dependent on the experimental condition.

It appears likely that one limiting factor in elucidating the mechanism of action of the glycoside has been the lack of means for direct study of the metabolic responses of the heart beating in a more physiological environment. By the technique of coronary sinus intubation, a direct study of patients treated with therapeutic doses of a rapid acting cardiac glycoside is possible. Simultaneous sampling of arterial and coronary venous blood, together with measurement of the coronary flow, permits the determination of the net myocardial gain or loss of foodstuffs, gases or other metabolites (11-13). While providing no information regarding intermediary reactions, data obtained by

1 Work supported by the U. S. Public Health Service Grant No. H-1129 (C3), The Life Insurance Medical Research Fund, the American Heart Association, and Sandoz Pharmaceuticals. this method should aid in elucidating the metabolic pathways within the heart muscle; thus reactions determined by more precise in vitro methods could be re-evaluated in the light of data obtained on the human heart in vivo.

The various cardiac glycosides differ in time of onset and persistence of action. Lanatoside-C, the preparation used in this study, is a purified derivative of digitalis lanata which can be given intravenously. The onset of action depends on the dosage used; with average digitalizing doses of 1.2 to $1.6 \mathrm{mgm}$., the so-called "vagal effect" usually appears within 15 to 20 minutes, reaching a maximum in about 40 minutes. Weisberger and Feil (14), for example, reported their results in treating 17 cases of resistant paroxysmal auricular tachycardia. Sixteen cases reverted to normal sinus rhythm within 40 minutes, with an average response of 17.6 minutes. Barrow (15), in a series of 26 patients, found the average time for reversion to be 19 minutes. Nicholson (16) administered $1.6 \mathrm{mgm}$. of Cedilanid ${ }^{\circledR}$ to 22 subjects with auricular fibrillation whose rates were stabilized at 120 beats per minute. In all patients there was a drop of from 15 to 80 beats within 15 minutes, with an average decrease in heart rate of 24 beats. Stead, Warren, and Brannon (17) administered $1.6 \mathrm{mgm}$. of lanatoside- $\mathrm{C}$ intravenously to 22 patients with congestive failure. The first effect noticed was a fall in venous pressure which began in 5 to 10 minutes and continued for 30 to 60 minutes. Cardiac output, measured 60 to 70 minutes after the injection, showed an average rise of 1.6 liters per minute. This effect was noted regardless of the cardiac rhythm or the initial stroke volume.

The present paper reports on the action of lanatoside-C on the myocardial metabolism of glucose, pyruvate, lactate, amino acids, ketones and 
fatty acids. A subsequent report will deal with the effect of this glycoside on the sodium and potassium balance of the human heart.

\section{PROCEDURE}

Twelve undigitalized adult patients were selected for the study (Table I). Seven of these had heart disease with evidence of left ventricular hypertrophy but had no symptoms of congestive failure other than decreased exercise tolerance. Five of these seven were hypertensive (J. B. J., G. G., E. E., W. H., and M. T.), one had rheumatic heart disease (D. T.) and one had aortic insufficiency due to syphilis (F. M.). Congestive heart failure was present in four patients. Heart failure in these individuals resulted from rheumatic valvular disease (L. W.), hypertension (W. B.) and from congenital malformation of the heart (E. F.). In the fourth patient the cause of the failure was unknown (J. R.). One of the subjects in the series had no evidence of heart disease (J. W.).

All patients were studied in the post adsorptive state. In three of the subjects (W. B., E. F., and J. R.) with congestive failure preliminary treatment with bed rest, diuretics and salt restriction was necessary before the studies could be performed. Small doses of merperidine or pentobarbital were given on the morning of the study to those individuals who seemed unduly apprehensive. The majority received no premedication.

Cardiac catheterization was performed in the usual manner $(12,13)$. If diagnostic procedures were indicated, these were completed first. Cardiac output was measured by the method of Fick (18), the mixed venous sample being obtained from the most distal cardiac chamber entered. Expired air was collected in a Douglas bag or Tissot spirometer. Oxygen and carbon dioxide in expired air was determined by the method of Scholander (19). The coronary sinus was intubated according to previously described methods (20). Simultaneous blood samples were collected from the coronary sinus and right femoral artery for determinations of oxygen, carbon dioxide, glucose, pyruvate, lactate, fatty acids, amino acids and ketones. In most cases duplicate samples were drawn and the results of the two analyses averaged. Coronary flow was measured 30 to 40 minutes following the ingestion of the glycoside. Coronary flow was not determined prior to the injection of lanatoside- $C$, since this would have unduly prolonged the procedure; also it had been previously found that digitalis in therapeutic doses does not influence the rate of coronary blood flow (21).

Lanatoside-C was administered intravenously in doses of .02 to $.03 \mathrm{mgm}$. per kil body weight, and coronary blood flow was measured using the nitrous oxide desaturation method $(22,23)$. Thirty to forty minutes later, or as soon as definite electrocardiographic changes appeared (slowing of heart rate, depression of ST segment or premature ventricular contractions), blood samples for metabolic determinations were again obtained from the femoral artery and coronary sinus.

Oxygen and carbon dioxide contents of blood were determined by the manometric method of Van Slyke and Neill (24). Blood glucose was measured by the method of Hagedorn and Jensen, using Somogyi's method to prepare the filtrates $(25,26)$. Pyruvate was determined according to the method of Friedemann and Haugen (27), using a tricloracetic acid filtrate, and lactate by the method of Barker and Summerson (28). For the determination of fatty acids the method of Man and Gildea was used (29). This is essentially a modification of the procedure of Stoddard and Drury's volumetric analysis (30). Amino acids were estimated according to the method of Albanese and Irby (31) and ketones by a modification of the micromethod of Greenberg and Lester (32).

From the analysis of the blood samples the myocardial extraction (coronary arteriovenous difference) of oxygen, carbon dioxide and individual metabolites was determined. The myocardial usage of 100 grams of left ventricle was calculated as the product of the myocardial extraction times the coronary flow. The oxygen extraction ratio was determined by multiplying the myocardial extraction by the oxygen equivalent of the particular metabolite divided by the myocardial oxygen extraction. This ratio represents the percentage of oxygen extracted by the heart which could be accounted for by complete catabolism of foodstuff to carbon dioxide and water. Because of the temporary storage of metabolites and their conversion within the metabolic pool, the sum of the individual oxygen extraction ratios at any one moment need not total 100 per cent.

The percentage myocardial extraction represents the percentage of the arterial concentration of a metabolite which is removed from coronary blood during its passage through heart muscle; it is calculated by the formula : $\frac{A-V}{A} \times 100$, where $A$ and $V$ represent the respective arterial and coronary venous concentrations of the metabolites. It has previously been shown that extraction of a substrate by the human heart varies in proportion to the arterial concentration (11). Since the percentage myocardial extraction takes into consideration the fluctuations in arterial level, this calculation provides a more accurate means of comparing changes in uptake of carbohydrates, fats and proteins by the myocardium than does the arteriovenous difference alone.

Evidence of Cedilanid@ effect was obtained from comparisons of electrocardiograms taken before and at intervals of five to ten minates following the injection of the glycoside. The earliest and most consistent change observed was slowing of the ventricular rate, which was present in nine subjects by the end of thirty minutes and was most marked in the two patients with auricular fibrillation (L. W. and E. F.). In seven subjects with normal sinus rhythm the average decrease amounted to 10 beats per minute. Other electrocardiographic changes included depression of the S-T segment in three patients, flattening of the $T$-waves in five patients, and the onset of frequent ventricular extrasystoles after digitalis administration in one individual. No electrocardiographic alterations were present in one patient, and the tracings of one subject were lost. However, the development of 
J. M. BLAIN, E. E. EDDLEMAN, A. SIEgEL, AND R. J. BING

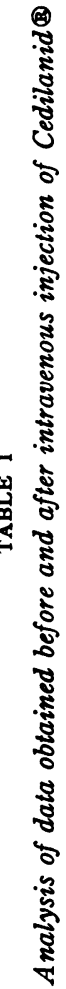

\begin{tabular}{|c|c|c|c|c|c|c|c|c|c|c|c|c|c|}
\hline 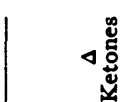 & 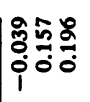 & 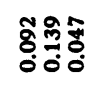 & 111 & च유 & 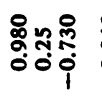 & 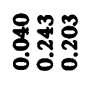 & 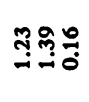 & స్๋ & 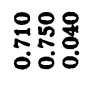 & วิธี่อ & 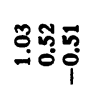 & 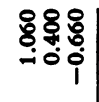 & 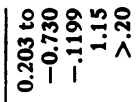 \\
\hline 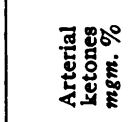 & 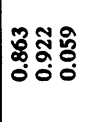 & 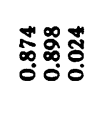 & 111 & & 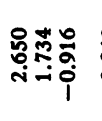 & $\begin{array}{l}\text { के कें } \\
\text { कें०० }\end{array}$ & जิ & 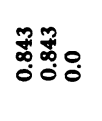 & 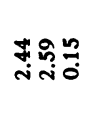 & 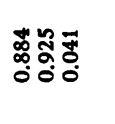 & \&્థి & 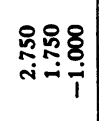 & 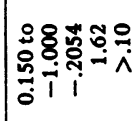 \\
\hline 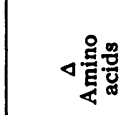 & $=$ & 808 & ĩ & కั:0ั & సีํํำ & $\therefore: 00$ & 111 & : & 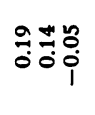 & 웅ㅎํㅇํํ & 111 & 011 & 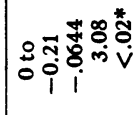 \\
\hline 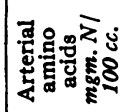 & î & 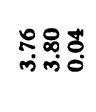 & 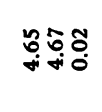 & $\begin{array}{l}\text { Nㅗㅇ } \\
\text { ming }\end{array}$ & 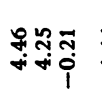 & 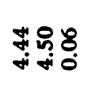 & 111 & 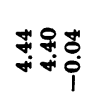 & 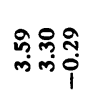 & ํำ & 111 & $\stackrel{m}{i}_{i} \mid 1$ & 웡 \\
\hline ব证总 & nᄄ & 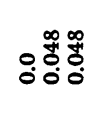 & 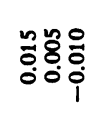 & 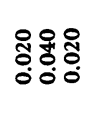 & 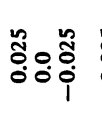 & 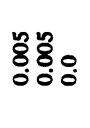 & 111 & 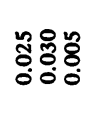 & $\begin{array}{l}n \\
0 \\
0 \\
0 \\
0\end{array} 0$ & \&: & 윰융유. & $\overbrace{0}^{\infty} \mid 1$ & 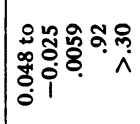 \\
\hline 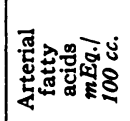 & (2) & 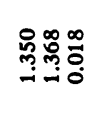 & 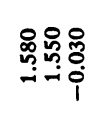 & 우ㄱㅠㅠㅇㅠ. & 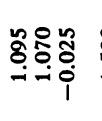 & 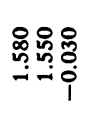 & 111 & 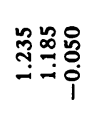 & 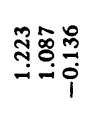 & 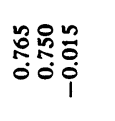 & 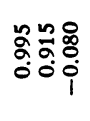 & 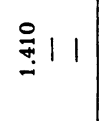 & 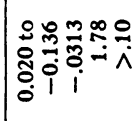 \\
\hline ه芯 & 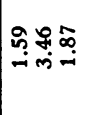 & 可兽品 & 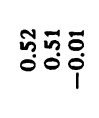 & mîn & 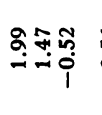 & 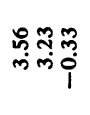 & 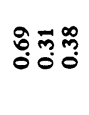 & 政 & 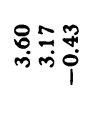 & 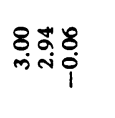 & 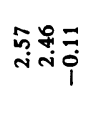 & 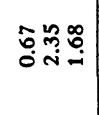 & 隹 \\
\hline 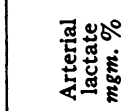 & 꾺유: & 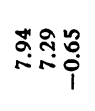 & 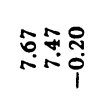 & 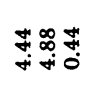 & 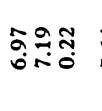 & 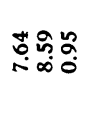 & 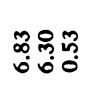 & 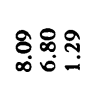 & Hân & 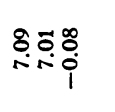 & 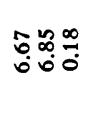 & 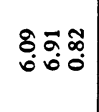 & 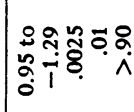 \\
\hline 唠 & 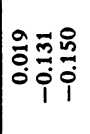 & 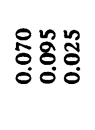 & 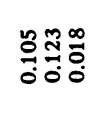 & 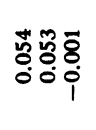 & 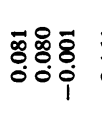 & î & 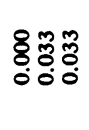 & 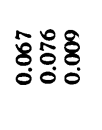 & 象解审 & 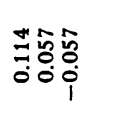 & 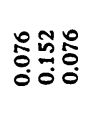 & 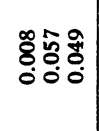 & i \\
\hline 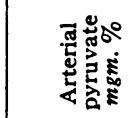 & 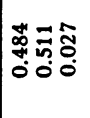 & 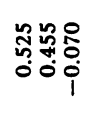 & 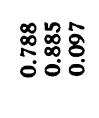 & 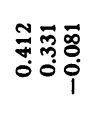 & 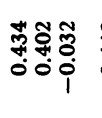 & 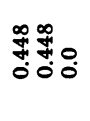 & 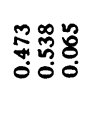 & 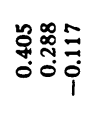 & 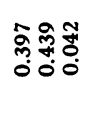 & 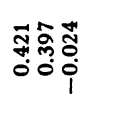 & 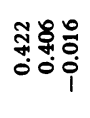 & 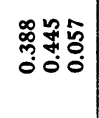 & ị \\
\hline ه: & OQ⿻日禸 & Fึ่ง & $\vec{i} \underset{\dot{m}}{\min }$ & 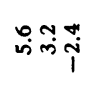 & 111 & Fஸ் & 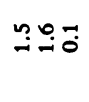 & $\stackrel{\infty}{~}$ & 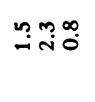 & ạ:å & 970 & $\stackrel{\infty}{\infty} \stackrel{0}{0}$ & 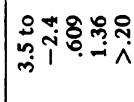 \\
\hline 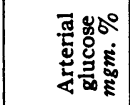 & 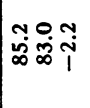 & 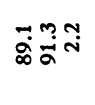 & ํํㅅํำ & 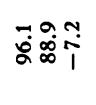 & 111 & 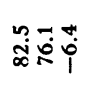 & مُ户் & सेंकें & ํํำำำ & ڤุ: & $\vec{\infty}$ & 열우 & \\
\hline 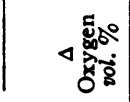 & ב־ & 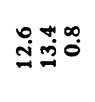 & $\stackrel{\sim}{0}||$ & 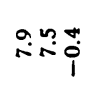 & $\stackrel{\infty}{\infty} \overbrace{0}^{m}: 0$ & جृi & mö. & $\stackrel{\infty}{m} \mid 1$ & 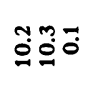 & mon & i̦ & $\stackrel{0}{\dot{i}} \stackrel{+}{\dot{m}}$ & 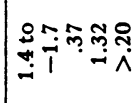 \\
\hline 列 & in & $\approx$ & $\Xi$ & $\stackrel{m}{=}$ & $\overline{0}$ & 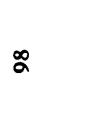 & 1 & 1 & 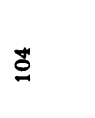 & $\ddot{n}$ & $\approx$ & 8 & \\
\hline 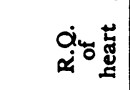 & $?$ & 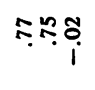 & $\stackrel{1}{1}$ & ஸุढั & 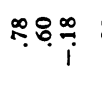 & סִ? & ำนุด & ọ। & 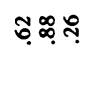 & 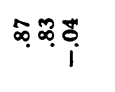 & ๓ạ: & కุ̣ & : \\
\hline 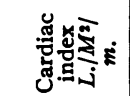 & 冓 & $\underset{m}{3}$ & a & $\stackrel{\leftrightarrow}{\dot{m}}$ & $\stackrel{P}{\dot{m}}$ & $\stackrel{n}{i}$ & $\underset{\infty}{\infty}$ & $\stackrel{\check{c}}{i}$ & $\stackrel{n}{n}$ & $\underset{i}{\stackrel{3}{i}}$ & $\stackrel{0}{0}$ & స్లి & \\
\hline & 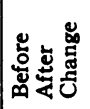 & 这䓌 & 产芯芯 & 这总 & 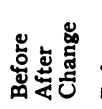 & 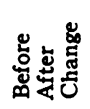 & 递总 & 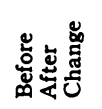 & 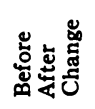 & 怤总 & 聯 & 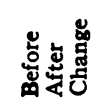 & 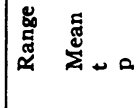 \\
\hline 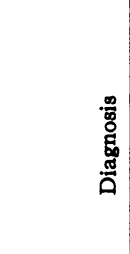 & 号 & 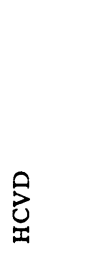 & 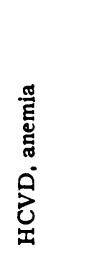 & 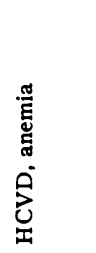 & 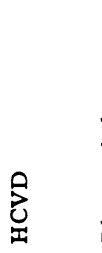 & 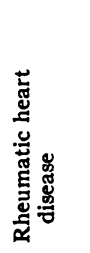 & 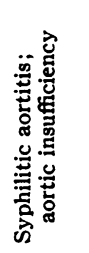 & 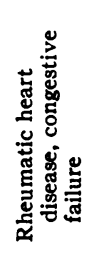 & 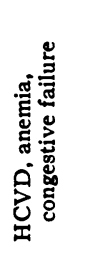 & 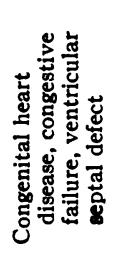 & 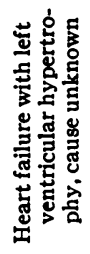 & 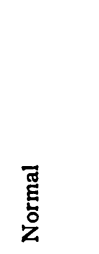 & \\
\hline ڤ̆ & $\Sigma$ & is & 5 & is & 4 & $\Sigma$ & $\Sigma$ & sut & $\Sigma$ & $\Sigma$ & $\Sigma$ & $\Sigma$ & \\
\hline$\stackrel{0}{4}$ & ลิ & $\stackrel{\infty}{+}$ & 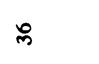 & $\neq$ & 8 & ले & $\stackrel{\infty}{\sim}$ & $\bar{m}$ & $\infty$ & $\infty$ & F & $\stackrel{\infty}{\circ}$ & \\
\hline$\stackrel{\overrightarrow{\mathrm{E}}}{\mathrm{E}}$ & $\therefore$ & $\begin{array}{l}\dot{0} \\
\dot{0}\end{array}$ & 球 & $\dot{3}$ & $\dot{\dot{\Sigma}}$ & $\dot{\dot{\theta}}$ & $\dot{\Sigma}$ & $\dot{3}$ & $\begin{array}{l}\dot{m} \\
\dot{3}\end{array}$ & 店 & $\dot{\sim}$ & $\dot{s}$ & \\
\hline i & - & N & $m$ & + & in & ○ & r & $\infty$ & $a$ & 은 & $=$ & $\simeq$ & \\
\hline
\end{tabular}




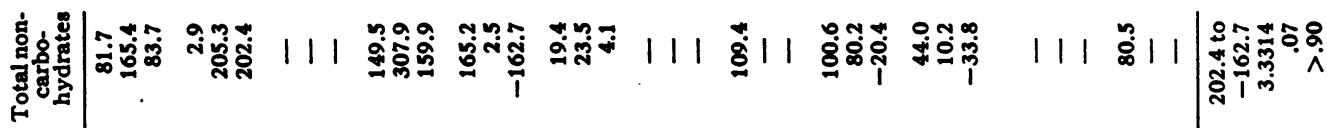

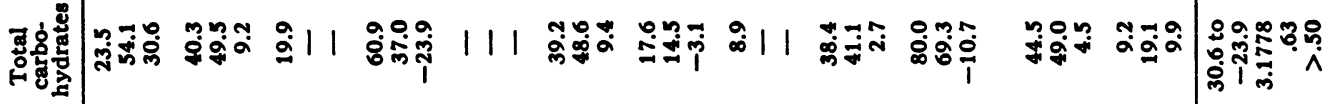

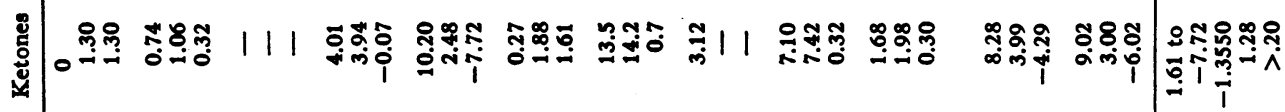

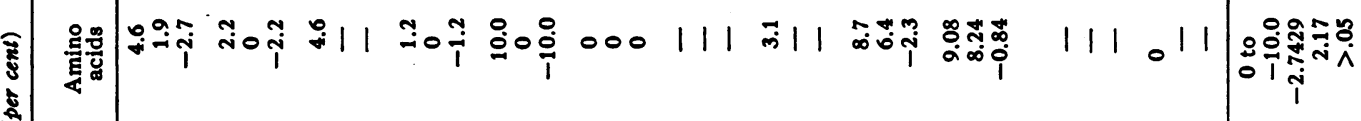

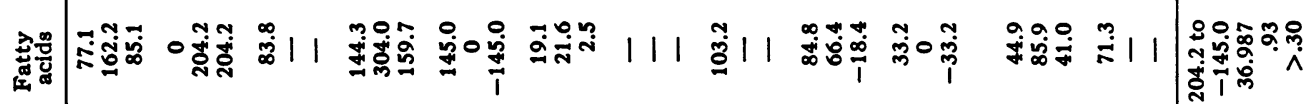

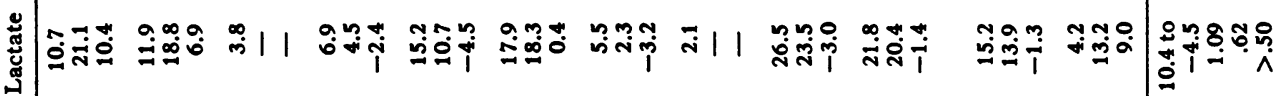
席产

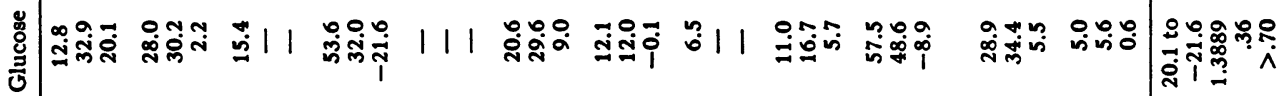

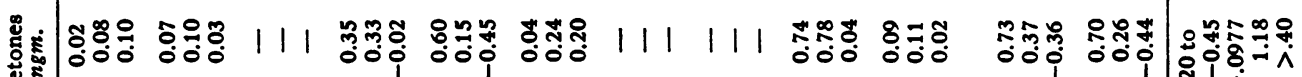

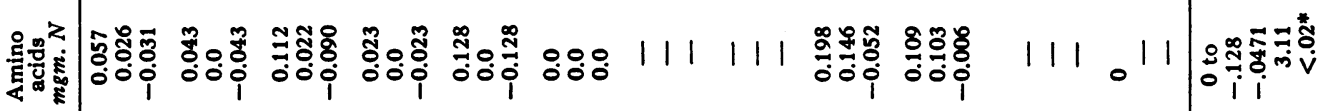

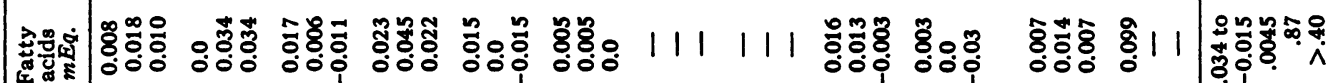

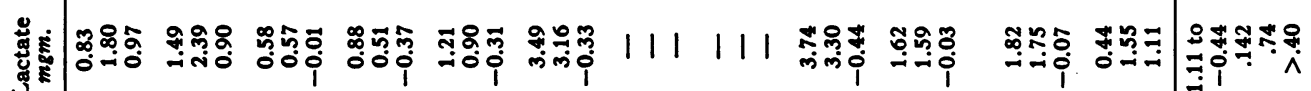
户่

造

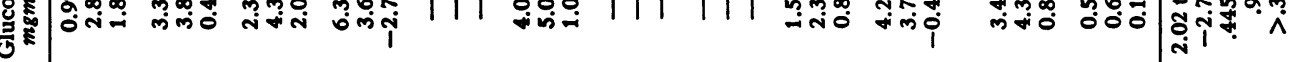
茖

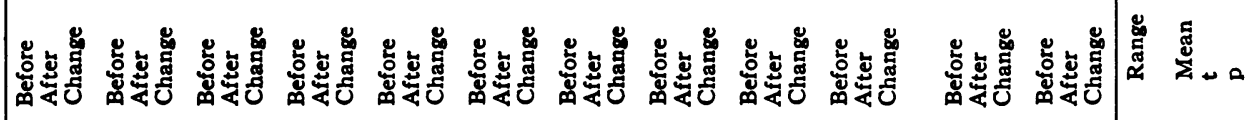

兽

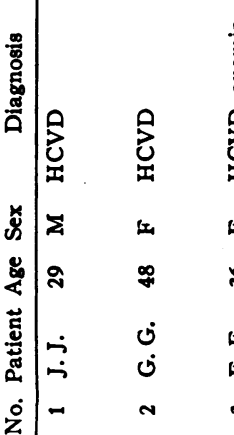

\begin{tabular}{|c|c|c|c|c|}
\hline 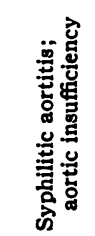 & 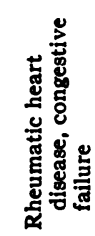 & 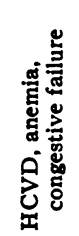 & 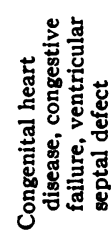 & 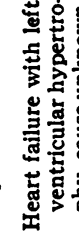 \\
\hline$\Sigma$ & 14 & $\Sigma$ & $\Sigma$ & $\Sigma$ \\
\hline $\boldsymbol{q}$ & $\bar{m}$ & $\infty$ & $\infty$ & $\approx$ \\
\hline ì & $\dot{3}$ & $\begin{array}{l}\dot{\infty} \\
\dot{3}\end{array}$ & 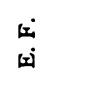 & $\ddot{\sim}$ \\
\hline - & $\infty$ & $a$ & 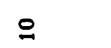 & $=$ \\
\hline
\end{tabular}


nausea and vomiting forty minutes after the administration of Cedilanide (ten minutes after blood samples were taken) probably constitutes adequate evidence of drug effect in this person.

The individual observations before and after Cedilanide administration are shown in Table I. Differences observed after injection of the glycoside were subjected to statistical analysis, each patient serving as his own control. Changes were considered significant only if there was less than one chance in twenty that the effect could have been due to chance alone $(p<.05)$.

\section{RESULTS}

No significant changes in the respiratory quotient of the heart could be attributed to the action of Cedilanid ${ }^{\circledR}$. Of the ten observations available before and after treatment, the respiratory quotient decreased in seven and increased in three (Table I). The myocardial oxygen extraction for the group showed a mean increment of 0.37 volumes per cent, increasing in eight subjects and decreasing in two. The difference was not statistically significant $(p>.20)$. Since digitalis causes no significant alteration in coronary flow, it can be assumed that myocardial oxygen usage likewise remained unchanged (21).

Observations of the arterial concentration and coronary arteriovenous glucose difference were available in eleven subjects. The arterial glucose concentration decreased in eight and increased in three patients, while the myocardial extraction de-

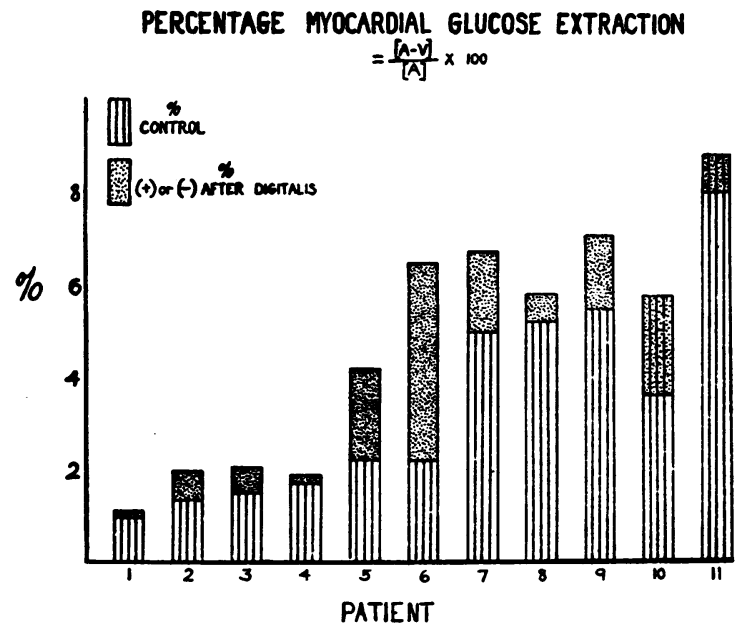

Fig. 1. Illustrates the Percentage Myocardial Glucose Extraction in the Eleven Patients Studied

This ratio increased in all but two patients (10 and $11)$; these had the highest initial percentage of glucose extraction. creased in two subjects and increased in nine. For the group as a whole, there was an average fall of $1.9 \mathrm{mgm}$. per $100 \mathrm{cc}$. in the arterial level of glucose and an average rise of $0.6 \mathrm{mgm}$. per $100 \mathrm{cc}$. in its myocardial extraction. Neither of these changes was statistically significant. Because of the lower arterial level, the percentage glucose extraction by the heart showed a more conspicuous gain, increasing from 3.7 per cent to 4.5 per cent. Although the $\mathrm{p}$ value was $>.10$, slightly above the level accepted as significant in this study, it can be seen in Figure 1 that the percentage extraction increased in all subjects except the two who initially had the highest percentages of glucose extraction (W. H. and E. F.).

Myocardial pyruvate extraction showed small fluctuations in both directions, but no consistent changes occurred which could be ascribed to the drug. There was a slight gain from 16.4 per cent to 19.2 per cent in the mean percentage pyruvate extraction. However, in four individual observations this ratio declined.

Alterations in arterial lactate level and myocardial lactate extraction were similarly inconsistent. As in the case of glucose and pyruvate the mean percentage extraction advanced slightly, rising from a control level of 24 per cent to 29 per cent after the administration of Cedilanid ${ }^{\circledR}$.

Data of fatty acid metabolism were available in ten subjects. The arterial concentration of fatty acids decreased in seven and increased in three individuals with a mean increase of $.031 \mathrm{mEq}$. per 100 cc. $(p>.10)$. Changes in fatty acid extraction and percentage extraction were more variable and did not approach statistical significance. The average percentage extraction by the heart increased from 1.2 per cent to 1.7 per cent. Despite the small percentage of available fatty acids extracted, their importance as a potential source of energy to the heart muscle is demonstrated by their high oxygen extraction ratios. In several instances the quantity of fatty acids removed from the coronary circulation could account for more than 100 per cent of the simultaneous oxygen extraction if it is assumed that they are completely metabolized to carbon dioxide and water. Similar findings have been described in previous publications $(11,13)$.

In eight of nine patients on whom data were available the amino acid extraction by the heart 
declined. In one subject myocardial extraction of amino acids was zero before and after injection of Cedilanid ${ }^{\circledR}$, and in four others the extraction fell to zero. It should be noted, however, that the arterial amino acid concentration also declined in six of the patients, so that the decrease in percentage extraction was not as marked as was the coronary arteriovenous difference. The mean percentage extraction before and after therapy was 2.5 per cent and 1.1 per cent, respectively. The myocardial metabolism of ketones was not altered by digitalis.

\section{DISCUSSION}

The mass of conflicting data in the literature makes it likely that the difference in metabolic effects of digitalis on heart muscle may be the result of various concentrations of drug employed and the differences in the kind of substrates and the techniques used in the studies $(10,33,34)$. However, even with identical techniques and concentrations of drugs the results have been contradictory. For example, various observers have noted that Ouabain increased the oxygen consumption of cardiac muscle slices respiring in the Warburg apparatus, while others have reported a decreased oxygen uptake with the same concentration of glycosides $(10,33)$. Using human auricular appendages obtained at operation, Burdette (35) found that the addition of lanatoside-C tripled the rate of respiration. David (36) on the other hand, found that after an initial increase Ouabain caused a diminution in the respiration of the isolated frog's auricle.

Studies on the heart-lung preparation have yielded equally variable results. Evans (37) reported that while digitalis increased cardiac output and efficiency, the oxygen consumption of the heart diminished. Peters and Visscher (9) found that digitalis increases both oxygen consumption and efficiency of the perfused heart. However, the increase in efficiency on a percentile basis was four to five times as great as the increase in myocardial oxygen consumption (9).

The bulk of evidence suggests that in the various types of experimental "failure" digitalis increases the work performance of the heart out of proportion to the changes in oxygen consumption, thereby increasing myocardial efficiency. In patients with congestive failure cardiac glycosides increase car- diac output and efficiency without causing significant changes in oxygen consumption (21).

The results of the present study demonstrate that digitalis in therapeutic dosage produces only minimal changes in the extraction of foodstuffs by the heart. Since there is some rise in the mean extraction percentage of pyruvate and lactate, the lower amino acid extraction after digitalis may be due to the "protein sparing" effect of carbohydrates.

The negative effect of Cedilanid ${ }^{\text {(b) }}$ on carbohydrate metabolism in the intact human heart contrasts with results of Wollenberger (34). This investigator found, in studying the action of Ouabain on the respiration of heart slices in the Warburg apparatus, that the glycoside accelerates the oxidation of glucose and lactate. This action accounts for the simultaneous increase in respiratory activity, with the acceleration of glucose oxidation exceeding, in proportion, that of the oxygen uptake. The results reported in this paper demonstrate only a slight elevation in percentage glucose extraction without any change in myocardial oxygen utilization. Since myocardial lactate utilization remains unchanged, a digitalis-induced shift from a glycolytic to a largely respiratory metabolism of carbohydrates as suggested by Wollenberger is unlikely in the human heart under these conditions.

The finding that the cardiac glycoside produces no significant changes in myocardial oxygen consumption or in total foodstuff utilization suggests that the improvement in work capacity of the failing heart induced by these glycosides must be the result of their action on energy liberation, or more specifically, on the contractile proteins of failing heart muscle directly. Digitalis can accelerate the spiraling of myosin threads (38). Mallov and Robb (39) reported that actomyosin threads prepared from beef hearts showed increased spiraling and shortening when digitoxin was added. In a later report the same authors, using protein surface films, found that Ouabain increased the work performance of actomyosin prepared in this manner (40). Digitalis also influences the polymerization of actin (41). Furthermore, digitoxin does not combine with sarcosomes of heart muscle, in which the enzymes concerned with energy production are concentrated (42). Additional evidence that cardiac glycosides do not influence energy 
production is available from the findings of Wollenberger (43) that Ouabain or Digoxin in therapeutic dosages causes no change in the concentration of high-energy phosphate carriers and phosphocreatine. Similar conclusions can be drawn from the work of Rothlin, Taeschler, and Cerletti (44). These investigators induced heart failure in the heart-lung preparation with dinitrophenol, a drug which increased myocardial oxygen consumption. Lanatoside- $C$ restored the initial work performance of the heart without further influencing myocardial oxygen consumption.

\section{SUMMARY}

The effects of therapeutic doses of Cedilanid ${ }^{\circledR}$ on the metabolism of glucose, pyruvate, lactate, amino acids, fatty acids and ketone bodies of the human heart have been investigated. The studies were carried out on patients using intubation of the coronary sinus.

Only minimal changes in the extraction of metabolites by the heart were observed. The findings suggest that the improvement in work capacity of the failing heart induced by the glycoside is the result of its action on energy liberation.

\section{REFERENCES}

1. Lown, B., and Levine, S., Current concepts in digitalis therapy. New England J. Med., 1954, 250, 771.

2. Stutz, H., Feigelson, E., Emerson, J., and Bing, R. J., The effect of digitalis (Cedilanid) on the mechanical and electrical activity of extracted and nonextracted heart muscle preparations. Circulation Res., 1954, 2, 555.

3. Hellems, H. K., Regan, T. J., and Talmers, F. N., Influence of acetyl strophanthidin on myocardial electrolyte exchange. J. Clin. Invest., 1955, 34, 915.

4. Wedd, A. M., The influence of digoxin on the potassium content of heart muscle. J. Pharmacol. \& Exper. Therap., 1939, 65, 268.

5. Wood, E. H., and Moe, G. K., Electrolyte and water content of the ventricular musculature of the heart-lung preparation with specific reference to the effects of cardiac glycosides. Am. J. Physiol., 1942, 136, 515.

6. Hagen, $P$. S., The effects of digilanid $C$ in varying dosage upon the potassium and water content of rabbit heart muscle. J. Pharmacol. \& Exper. Therap., 1939, 67, 50.

7. Calhoun, J. A., and Harrison, T. R., Studies in congestive heart failure. IX. The effect of digitalis on the potassium content of the cardiac muscle of dogs. J. Clin. Invest., 1931, 10, 139.
8. Boyer, P. K., and Poindexter, C. A., The influence of digitalis on the electrolyte and water balance of heart muscle. Am. Heart J., 1940, $20,586$.

9. Peters, H. C., and Visscher, M. B., The energy metabolism of the heart in failure and the influence of drugs upon it. Am. Heart J., 1936, 11, 273.

10. Wollenberger, A., The energy metabolism of the failing heart and the metabolic action of the cardiac glycosides. Pharmacol. Rev., 1949, 1, 311.

11. Bing, R. J., The metabolism of the heart. Harvey Lecture Series (In Press).

12. Bing, R. J., Siegel, A., Vitale, A. G., Balboni, F., Sparks, E., Taeschler, M., Klapper, M., and Edwards, S., Metabolic studies on the human heart in vivo. I. Studies on carbohydrate metabolism of the human heart. Am. J. Med., 1953, 15, 284.

13. Bing, R. J., Siegel, A., Ungar, I., and Gilbert, M., Metabolism of the human heart. II. Studies on fat, ketone and amino acid metabolism. Am. J. Med., 1954, 16, 504.

14. Weisberger, A. S., and Feil, H., Lanatoside $C$ in the treatment of persistent paroxysmal auricular tachycardia. Am. Heart J., 1947, 34, 871.

15. Barrow, J. G., Treatment of paroxysmal supraventricular tachycardia with Lanatoside C. Ann. Int. Med., 1950, 32, 116.

16. Nicholson, J. A., The intravenous use of lanatoside C. New England J. Med., 1943, 229, 619.

17. Stead, E. A., Jr., Warren, J. V., and Brannon, E. S., Effect of lanatoside $C$ on the circulation of patients with congestive failure. A study using catheterization of the right side of the heart. Arch. Int. Med., 1948, 81, 282.

18. Fick, A., Ueber die Messung des Blutquantums in den Herzventrickeln. Verhandlungen. Sitzungsbericht, phys. med. Gesell. zu Würzburg, N.F. 2, Jul. 1870, p. XVI.

19. Scholander, P. F., Analyzer for accurate estimation of respiratory gases in one-half cubic centimeter samples. J. Biol. Chem., 1947, 167, 235.

20. Bing, R. J., Vandam, L. D., Gregoire, F., Handelsman, J. C., Goodale, W. T., and Eckenhoff, J. E., Catheterization of the coronary sinus and the middle cardiac vein in man. Proc. Soc. Exper. Biol. \& Med., 1947, 66, 239.

21. Bing, R. J., Maraist, F. M., Dammann, J. F., Jr., Draper, A., Jr., Heimbecker, R., Daley, R., Gerard, $R$, and Calazel, P., Effect of strophanthus on coronary blood flow and cardiac oxygen consumption of normal and failing human hearts. Circulation, 1950, 2, 513.

22. Spencer, F. C., Merrill, D. L., Powers, S. R., and Bing, R. J., Coronary blood flow and cardiac oxygen consumption in unanesthetized dogs. Am. J. Physiol., 1950, 160, 149.

23. Goodale, W. T., and Hackel, D. B., Measurement of coronary blood flow in dogs and man from rate of myocardial nitrous oxide desaturation. Circulation Res., 1953, 1, 502. 
24. Van Slyke, D. D., and Neill, J. M., The determination of gases in blood and other solutions by vacuum extraction and manometric measurement. J. Biol Chem., 1924, 61, 523.

25. Hagedorn, H. C., and Jensen, B. N., Zur Mikrobestimmung des Blutzuckers mittels Ferricyanid. Biochem. Ztschr., 1923, 135, 46.

26. Somogyi, M. A., Method for the preparation of blood filtrates for the determination of sugar. J. Biol. Chem., 1930, 86, 655.

27. Friedemann, T. E., and Haugen, G. E., Pyruvic acid. II. The determination of keto acids in blood and urine. J. Biol. Chem., 1943, 147, 415.

28. Barker, S. B., and Summerson, W. H., The colorimetric determination of lactic acid in biological material. J. Biol. Chem., 1941, 138, 535.

29. Man, E. B., and Gildea, E. F., A modification of the Stoddard and Drury titrimetric method for the determination of the fatty acids in blood serum. J. Biol. Chem., 1932, 99, 43.

30. Stoddard, J. L., and Drury, P. E., A titration method for blood fat. J. Biol. Chem., 1929, 84, 741.

31. Albanese, A. A., and Irby, V., Determination of amino nitrogen of blood filtrates by the copper method. J. Lab. \& Clin. Med., 1945, 30, 718.

32. Greenberg, L. A., and Lester, D. A., Micromethod for the determination of acetone and ketone bodies. J. Biol. Chem., 1944, 154, 177.

33. Olson, R. E., and Schwartz, W. B., Myocardial metabolism in congestive heart failure. Medicine, 1951, 30, 21.

34. Wollenberger, A., Metabolic action of the cardiac glycosides. III. Influence of ouabain on the utili- zation of C-14 labeled glucose, lactate, and pyruvate by dog heart slices. Arch. f. exper. Path. u. Pharmakol., 1953, 219, 408.

35. Burdette, W. J., Increase in oxygen consumption of human cardiac muscle incubated with lanatoside $\mathrm{C}$. J. Lab. \& Clin. Med., 1952, 40, 867.

36. David, J. C., The action of drugs on the oxygen consumption of the frog's isolated auricle. J. Pharmacol. \& Exper. Therap., 1930, 40, 229.

37. Evans, C. A. L., Metabolism of the heart. Edinburgh M. J., 1939, 46, 733.

38. Bowen, W. J., Effects of digoxin upon rate of shortening of myosin B threads. Federation Proc., 1952, $11,16$.

39. Mallov, S., and Robb, J. S., Behavior of actomyosin threads. Federation Proc., 1949, 8, 104.

40. Robb, J. S., and Mallov, S., Effect of ouabain on actomyosin threads. J. Pharmacol. \& Exper. Therap., 1953, 108, 251.

41. Horváth, I., Király, C., and Szerb, J., Action of cardiac glycosides on the polymerization of actin. Nature, 1949, 164, 792.

42. Friedman, M., and St. George, S., The cardiac and hepatic intracellular fate of digitoxin. J. Clin. Invest., 1953, 32, 569.

43. Wollenberger, A., Metabolic action of the cardiac glycosides. II. Effect of ouabain and digoxin on the energy-rich phosphate content of the heart. J. Pharmacol. \& Exper. Therap., 1951, 103, 123.

44. Rothlin, E., Taeschler, M., and Cerletti, A., Action of dinitrophenol and lanatoside $C$ on the canine heartlung preparation. Circulation Res., 1955, 3, 32. 